\section{How Good Are Our Best Models? Jackknifing, Bootstrapping, and
Earthquake Depth}

PAGES 593, 605-606

\section{Bart W. Tichelaar and Larry J. Ruff}

Department of Geological Sciences, University of Michigan, Ann Arbor

\section{Introduction}

Many techniques have been developed to extract a model from data. In general, these techniques are based on minimization of the misfit between measured data and predicted "data." The model is connected to the predicted "data" by a physical theory. To know how good the model is, one must evaluate model variance. Since the data variance, or alternatively the misfit, is generally nonzero model variance is generally nonzero. In many cases, the model is a linear function of the data, and model variance can be estimated by formally mapping the data variance to model space [e.g., Menke, 1984].

Estimation of data variance is a problem in itself. Because many geophysical data are nonreproducible (e.g., a certain earthquake cannot be repeated), it is not possible to directly verify any assumptions made with re. spect to the probability distribution of the data. A further difficulty in estimating model variance is that the physical relation between data and model is nonlinear in many problems. For these cases, it is often not possible to find an analytical expression for model variance in terms of data variance. Thus it is important to use methods that are insensitive to assumptions made with respect to the statistical properties of the data and that do not need an analytical expression for the connection between model variance and data variance.

Resampling techniques form a group of such statistical methods. Resampling techniques can be designed that are insensitive to the probability distribution of the data, e.g., each datum does not need to have equal vari-

\section{Cover.}

Southern California earthquakes light up not only faults (blue) but also folds (red) Some 24,000 events during 1980-1986 (yellow circles proportional to magnitude, $1.5 \leqslant M L \leqslant 6.5$ ) recorded on 700 seismometers concentrate along some portions of the San Andreas or in the Los Angeles Basin (through-going diagonal line) and other faults that can be seen at Earth's surface. But many earthquakes such as those parallel to the San Andreas or in the Los Angeles Basin (lower right corner), appear more closely bounds to folds, an association explicable if folds mark the location of active blind thrust and reverse faults.

This argument is advanced in "Hidden Earthquakes," appearing in the June 1989 ance (data may be heteroscedastic), nor do the data have to follow a normal distribution. Furthermore, these techniques allow evaluation of statistical properties that cannot be determined analytically.

Resampling techniques are based on notion that we can repeat the experiment by constructing multiple data sets from the one measured data set. Our presentation will focus on two techniques: jackknifing and bootstrapping. We do not intend to be complete; statisticians have written many technical papers about jackknifing and bootstrapping. Our goal is merely to promote the use of resampling techniques in geophysics. These techniques are easy to use and offer great promise for estimating the best model and model variance in linear and complicated nonlinear problems [e.g., McLaughlin, 1988; Guttorp and Walden, 1987; Willmott et al., 1985]. In fact, many scientists may already have applied some resampling technique to their data without realizing that it is a "legitimate" statistical technique. Jackknifing and bootstrapping are fields of current research in statistics, and some topics discussed in this paper are still controversial. The reader who wants to use a resampling method is advised to use the reference list for a more detailed description. The two main sources for this paper are $W u$ [1986] and Efron [1982]

\section{Resampling}

The key concept of jackknifing and bootstrapping is that the original data set is re sampled to form a large number of data sets and the subsequent multiple estimates of the model give information on model variance. Henceforth, we shall assume that both data

issue of Scientific American. In addition, a Special Section of the July 1989 issue of the Journal of Geophysical Research-Solid Earth and Planets will focus on the small but ominous $1987 \mathbf{M}=6$ Whittier Narrows, Calif. earthquake in the Los Angeles Basin (largest hexagon in the lower right) which struck beneath and uplifted the Santa Monica Mountains fold. The computer file containing the folds can be accessed via the United States Geological Survey's GSVAXO. Run we:[oppenheimer.map] sift. The folds are in the "special features" menu; each has a citation and a known or inferred age. (This map provided by John E. Estreem, Jerry P. Eaton, and Ross S. Stein of the United States Geological Survey, Menlo Park, Calif.) and model are discretized. A resample is a "copy" of the original data that may contain a certain original datum more than once, once, or not at all. To be more specific, suppose the model that is to be estimated can be represented by a set of $p$ model parameters $\left(\mathrm{m}_{1}, \ldots, \mathrm{m}_{\mathrm{p}}\right)^{\mathrm{T}}=\overline{\mathrm{m}}$, and the data can be written as a vector with $n$ components $\bar{d}=\left(d_{1}, \ldots, d_{n}\right)^{T}$, where $\mathrm{T}$ denotes the transpose. Suppose also that a physical theory connects each datum $d_{i}$ to the model with a mathematical function $f_{i}$ that is, $d_{i}=f_{i}(\bar{m})+\varepsilon_{i}$, where $\varepsilon_{i}$ represents the noise in the physical system. The model $\bar{m}$ is typically estimated by minimizing the squared distance between $\bar{d}$ and $\bar{f}$ with respect to the model, $\overline{\mathrm{m}}_{\mathrm{est}}=\min _{\overline{\mathrm{m}}}^{\overline{-}}\left[(\overline{\mathrm{d}}-\overline{\mathrm{f}}(\overline{\mathrm{m}}))^{\mathrm{T}}(\overline{\mathrm{d}}-\overline{\mathrm{f}}(\overline{\mathrm{m}}))\right]$. This least squares procedure may or may not produce a unique $\overline{\mathrm{m}}_{\text {est }}$. In the latter case, a unique $\overline{\mathrm{m}}_{\text {est }}$ can be produced by model damping. Let us now derive a new model estimate from a "new," i.e., resampled, data set. The resampled data set is a vector with $\mathrm{k}$ components. Note that $\mathrm{k}$ may be smaller than $\mathrm{n}$ because the resampled data vector may have fewer components than the original data. In com pact notation we can write the resampled data vector $\overline{\mathrm{d}}^{*}$ as

$$
\overline{\mathrm{d}}^{*}=\mathrm{D} \cdot \overline{\mathrm{d}}
$$

where the dot stands for matrix contraction. The matrix D, which we will refer to as the resampling operator, has $\mathrm{k}$ rows and $\mathrm{n}$ columns, and each row contains only one nonzero component. The value of this component is always 1 . Of course, for the model estimation we select $\overline{\mathrm{f}}^{*}(\overline{\mathrm{m}})$ such that it corresponds with $\bar{d}^{*}: \bar{f}^{*}=D \cdot \bar{f}$. Each resample defines a model estimate $\overline{\mathrm{m}}^{*}$ est $=$ $\min _{\bar{\pi}}\left[\left(\bar{d}^{*}-\bar{f}^{*}(\bar{m})\right)^{\mathrm{T}}\left(\bar{d}^{*}-\bar{f}^{*}(\overline{\mathbf{m}})\right)\right]$, and in a jackknife or bootstrap resampling scheme these multiple model estimates give information on the model variance. Jackknifing and bootstrapping both resample the data set but use a different resampling scheme. Technically, it comes down to a different choice and dimension of the resampling operator, which has direct consequences for the way in which the multiple model estimates are combined to calculate model variance.

\section{Jackknifing}

A jackknife resample is extracted from the original data by deleting a fixed number, say $\mathrm{j}$, of the $n$ data points (Figure 1). In other words, the resampling operator has $k=n-j$ rows and $n$ columns. Not only each row, but also each column, has only one nonzero component (with the value of 1 ), which implies that an original datum is never copied into a resample more than once. The total number of possible jackknife resamples and model es timates is $\left[\begin{array}{l}n \\ j\end{array}\right]$. How is model variance estimated from these multiple model estimates? Before we give a general expression for the variance estimator of the "delete-j" jackknife, we will follow Efron's [1982] example to illus trate how the variance of the sample average is obtained, using the "delete-1" jackknife $(j=1, k=n-1)$. Because most researchers are interested in standard deviation rather than variance, all expressions will be for the square root of variance. For a sample average, 


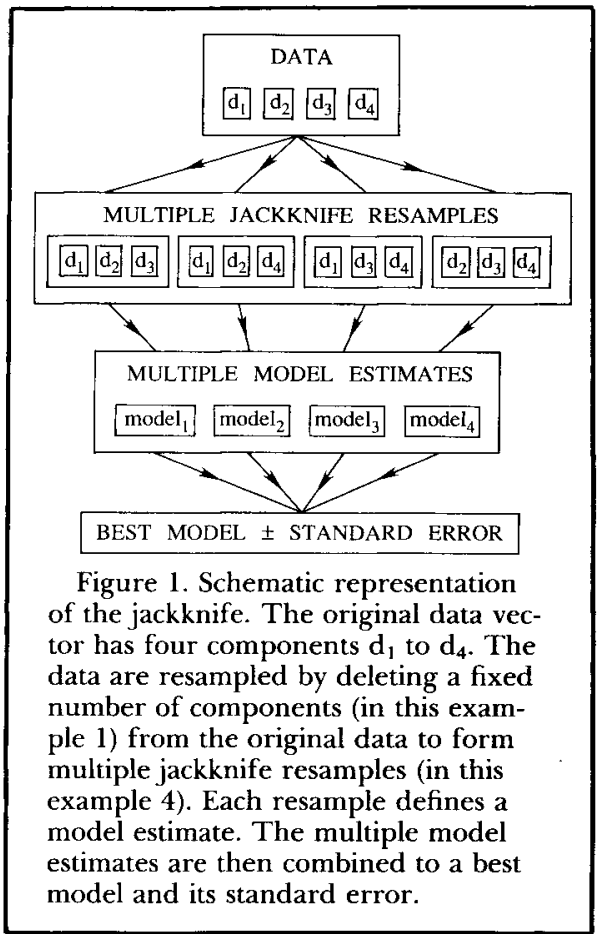

$$
\hat{\mathrm{x}}=\frac{1}{\mathrm{n}} \sum_{\mathrm{i}=1}^{\mathrm{n}} \mathrm{x}_{\mathrm{i}}
$$

the usual estimator for the standard deviation

$$
\hat{\sigma}=\left[\frac{1}{n(n-1)} \sum_{i=1}^{n}\left(x_{i}-\hat{x}\right)^{2}\right]^{1 / 2}
$$

For the sample average of the data set deleting the $i^{\text {th }}$ datum, we can write

$$
\hat{\mathbf{x}}_{i}^{*}=\frac{n \hat{\mathbf{x}}-\mathbf{x}_{i}}{n-1}=\frac{1}{n-1} \sum_{j \neq i} x_{j}
$$

This procedure will give $\left[\begin{array}{l}n \\ 1\end{array}\right]=\mathbf{n}$ jackknife averages $\hat{\mathrm{x}}_{\mathrm{i}}{ }^{*}$, with average

$$
\overrightarrow{\mathbf{x}}=\sum_{\mathbf{i}=1}^{\mathrm{n}} \frac{\hat{\mathbf{x}}_{\mathrm{i}}^{*}}{\mathrm{n}}
$$

Note that the numerical value of $\tilde{\mathrm{x}}=\hat{\mathrm{x}}$, i.e., the average of the jackknife averages equals the average of the full data set. It is easy to verify that the "delete-1" jackknife estimator of standard deviation $\hat{\sigma}_{J}$

$$
\hat{\sigma}_{J}=\left[\frac{n-1}{n} \sum_{i=1}^{n}\left(\hat{x}_{i}^{*}-\tilde{x}\right)^{2}\right]^{1 / 2}
$$

equals the usual expression (2). The advantage of (4) is that it can be generalized to an estimator of the standard deviation for any statistic $\theta$ that can be estimated from the data. Where $\theta$ is a scalar, this can simply be done by replacing $\hat{\mathbf{x}}_{i}{ }^{*}$ with $\hat{\boldsymbol{\theta}}_{i}{ }^{*}$ and $\tilde{\mathbf{x}}$ with $\tilde{\boldsymbol{\theta}}$, where $\hat{\boldsymbol{\theta}}_{i}^{*}$ is an estimator of $\theta$, calculated for the data set with the $\mathrm{i}^{\text {th }}$ datum deleted. A following step is then to study the properties of this estimator of the standard deviation of $\theta$. The smaller its bias, the closer its expectation to the true standard deviation. Furthermore, the estimator should be robust with respect to the statistical properties of the data. Efron and
Stein [1981] show that the "delete-1" jackknife variance estimator for a general scalar statistic, $\hat{\theta}\left(\mathrm{d}_{1}, \mathrm{~d}_{2}, \cdots, \mathrm{d}_{\mathrm{n}}\right)$, is biased upward (if biased) and thus gives a conservative estimate of the true variance. Their result is proven for independently, identically distributed data $d_{i}$. There is no special reason why only one sample should be deleted to form a jackknife resample. Furthermore, $W u$ [1986] remarks that if there is a good reason to believe that certain jackknife resamples give better model estimates than others, this should be accounted for in the estimator. He arrives at the following expression for the general weighted "delete-j" jackknife estimator of standard deviation $\hat{\sigma}_{J A C K}$.

$\hat{\sigma}_{\text {JACK }}=$

$\left[\frac{\mathrm{k}-\mathrm{p}+1}{\mathrm{n}-\mathrm{k}} \sum_{\mathrm{i}} w_{i}^{*}\left(\hat{\boldsymbol{\theta}}_{\mathrm{i}}^{*}-\tilde{\boldsymbol{\theta}}\right)^{\mathbf{T}}\left(\hat{\boldsymbol{\theta}}_{\mathrm{i}}^{*}-\overline{\boldsymbol{\theta}}\right)\right]^{1 / 2}$

where $k$ is the subset size $(k=n-j)$, p the number of model parameters, and the summation is over all the subsets of size $\mathrm{k}$. The statistic $\theta$ can be the model $\overline{\mathbf{m}}$ but can in general be any function of the data. For every subset estimate $\hat{\boldsymbol{\theta}}_{i}^{*}$, a weight $w_{i}^{*}$ accounts for the rela tive importance of the corresponding jackknife resample in the calculation of model variance. These weights are normalized as

$$
\sum_{i} w_{i}^{*}=1
$$

$W u$ [1986] gives the expression for $w_{i}^{*}$ and $\hat{\theta}_{i}^{*}$ for the case in which $\theta$ is a linear function of the data and shows that $\hat{\sigma}_{J A C K}$ is almost

unbiased for independently, identically distributed data, and that $\hat{\sigma}_{J A C K}$ is robust against data error variance heteroscedasticity for $k=n-1$. Wu also considers the case in which $\theta$ is a nonlinear function of the data.

To illustrate the use of the jackknife, we did a simple experiment in linear analysis, where analytical expressions for model variance are available: estimation of the variance of the slope of a straight line through the origin. The data (Figure 2a) consist of 20 unequally spaced points $y_{i}$, that follow the relation $\mathrm{y}_{\mathrm{i}}=\mathrm{cx}_{\mathrm{i}}+\varepsilon_{\mathrm{i}}(\mathrm{c}=1.5)$. Each noise component $\varepsilon_{\mathrm{i}}$ is drawn from a white distribution with a standard deviation of 1.5 and mean 0 . The linear least squares estimator for the standard deviation of the slope is

$$
\hat{\boldsymbol{\sigma}}=\left[\left(\sum\left(\mathrm{y}_{\mathrm{i}}-\hat{c} \mathrm{x}_{\mathrm{i}}\right)^{2}\right) /(\mathrm{n}-1) \sum \mathrm{x}_{\mathrm{i}}^{2}\right]^{1 / 2}
$$

where $\hat{c}=\Sigma x_{i} y_{i} / \Sigma x_{i}^{2}$. We calculated the standard error of the slope using two jackknife estimators, equation (5): (i) for the "delete-1" jackknife ( $k=n-1)$ and (ii) for the "deletehalf" jackknife $(k=n / 2)$. The jackknifes are unweighted $\left(w_{\mathrm{i}}^{*}=\right.$ constant for all (i)). For the "delete-half" jackknife we used a Monte Carlo evaluation of 100 resamples because the total number of resamples is very large. Figures $2 b$ and $2 c$ show the results. The estimated standard deviations agree with the analytic least squares value. An interesting aspect is the difference in scale of the frequency distribution for the two jackknifes (Figures $2 b, 2 c)$. This scale difference arises from the fact that for general $k$ the resampling error $\hat{\theta}_{\mathrm{i}}{ }^{*}-\tilde{\theta}$ has a different stochastic order than

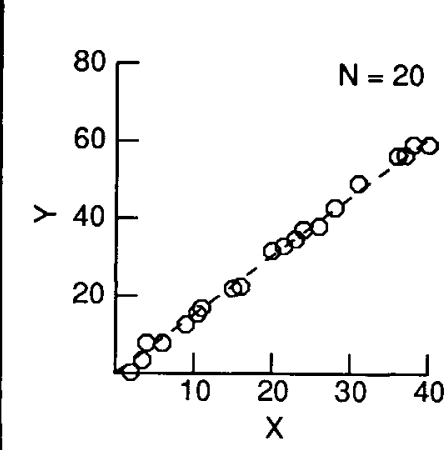

(a)

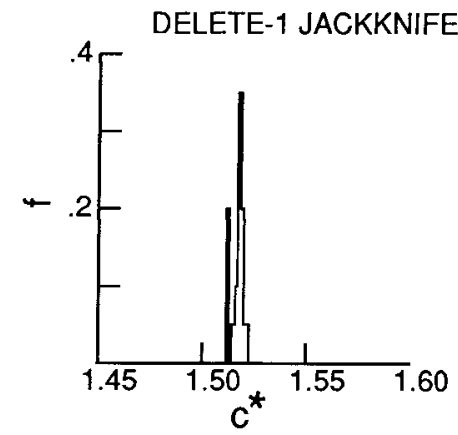

(b)

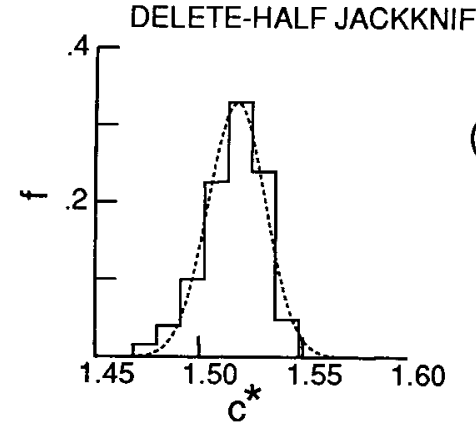

(c)

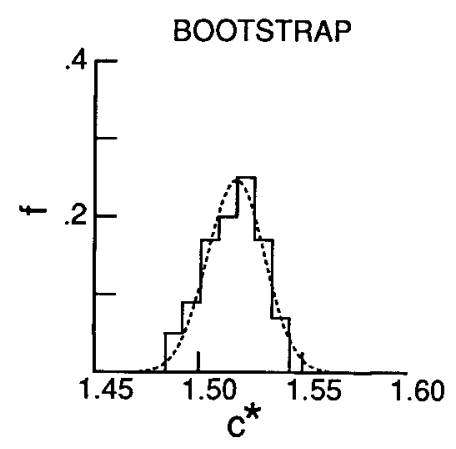

Fig. 2. (a) A least squares fit through the noisy data give a slope of $\hat{\mathbf{c}}=$

$1.518 \pm 0.0138$ ( 1 standard error). (b) The normalized frequency ( $f$ ) distribution for the "delete-1" jackknife yields an estimate of 1.518 \pm 0.0136 . (c) For the "delete-half" jackknife the value is $1.517 \pm 0.0141 .(d)$ Bootstrapping gives an estimated slope of $1.517 \pm 0.0132$. Note the scale difference between the distributions in $2 b$ and $2 c$ (see also formula (5)). The dashed line $(2 c, 2 d)$ represents the analytical distribution of $\hat{c}$. 
the sampling error $\hat{\theta}-\theta[W u, 1986]$. The scale difference is corrected by the scale factor $\sqrt{(k-p+1) /(n-k)}$ in expression (5). For the "delete-half" jackknife, this factor is 1 .

\section{Bootstrapping}

A bootstrap resample is a random selection of $\mathbf{n}$ data out of $\mathbf{n}$ original data (Figure 3 ). In contrast with the jackknife, the resampling operator is a square matrix and each column may contain more than one 1 , which means that a resample may contain a certain original datum more than once. Just like the jackknife, the bootstrap estimator of standard deviation $\hat{\sigma}_{B O O T}$ can be calculated without knowing an analytical expression that relates the statistic of interest with the data. Suppose that $\hat{\theta}_{i}^{*}$ is an estimator of the statistic $\theta$ of interest, calculated for the bootstrap resample $i$.

To do a Monte Carlo approximation of $\hat{\sigma}_{B O O T}$, a large number $\mathrm{L}$ of bootstrap estimators $\hat{\theta}_{i}{ }^{*}$ need to be calculated. The bootstrap estimate of the standard deviation of $\theta$ is

$$
\hat{\sigma}_{B O O T}=\left[\frac{1}{\mathrm{~L}-1} \sum_{\mathrm{i}=1}^{\mathrm{L}}\left(\hat{\theta}_{\mathrm{i}}^{*}-\tilde{\theta}\right)^{\mathrm{T}}\left(\hat{\theta}_{\mathrm{i}}^{*}-\tilde{\theta}\right)\right]^{1 / 2}
$$

with $\tilde{\theta}=\sum$

The summation is over all $L$ bootstrap samples. Figure $2 d$ shows the bootstrap evaluation for the straight-line example from the previous section $(\mathrm{L}=100)$. The bootstrap standard error of the slope is slightly smaller than both the jackknife estimates. Efron [1982], who introduced the bootstrap, shows that for the linear case with independently, identically distributed data, $\hat{\sigma}_{B O O T}$ is slightly downward biased. Just like the jackknife, there are different bootstrap estimators of standard deviation. The weighted bootstrap, bootstrapping of normalized residuals, the smoothed bootstrap, and the parametric

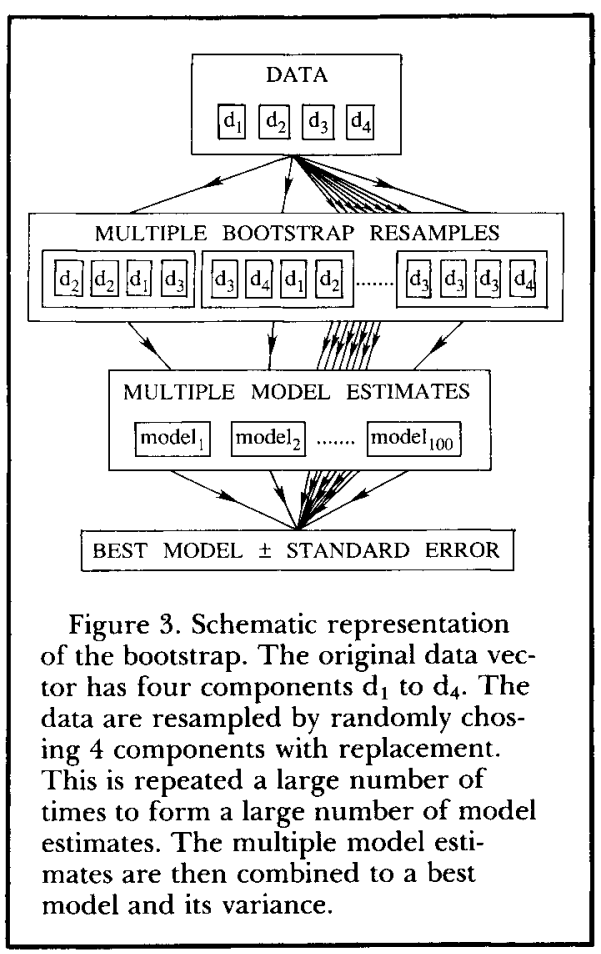

bootstrap are some examples. [Wu, 1986; Efron, 1979, 1982; Efron and Gong, 1983]. Whether one should use a jackknife or bootstrap estimator to estimate model variance, and what type of bootstrap or jackknife, depends on the particular problem, but also seems to be somewhat controversial (see, e.g., the discussions by several authors that are published together with $W u$ 's [1986] paper). The big advantage for all discussed resampling techniques is that they can be used to evaluate the statistical properties of a statistic that is related to the data in a complicated, nonanalytical way. Apart from estimation of standard deviation, resampling can be used to estimate mean, median, and even to construct confidence intervals. This completes our introductory discussion of jackknifing and bootstrapping. We now cross over to a seismological problem.

\section{Earthquake Depth}

Depth is a fundamental focal parameter of earthquakes. For example, the depth of large shallow earthquakes that occur on the interface between two plates in a subduction zone is of importance with respect to the study of seismic coupling [Tichelaar and Ruff, 1988]. Although routine determination of focal parameters may give adequate estimates of the epicentral coordinates and focal mechanism, depth estimates for shallow earthquakes may not be accurate enough for geophysical considerations of seismic coupling. We determine the best depth by $P$ wave inversion, where we match the direct $P$ and surface reflections of a set of well-distributed seismographic stations simultaneously [Ruff, 1989]. Focal depth is a nonlinear parameter in waveform inversion. The waveforms (seismic waves) are generated by the earthquake, propagate through the Earth, and are recorded by seismographs. For long-period $P$ waves the focal mechanism and propagational effects, as well as the system response of the seismograph can be characterized by a Green's function $G_{\mathrm{z}}(\mathrm{t})$ for a "unit impulse" earthquake at depth $z$. The seismic source time history can be represented by the moment rate function $\dot{M}(t)$, which is proportional to the fault-averaged displacement rate. A $P$ wave seismogram is simply the convolution of the Green's function with the moment rate function. For a set of $f$ seismograms $s^{(1)}(t), \ldots, s^{(f)}(t)$, the relation with depth and moment rate is
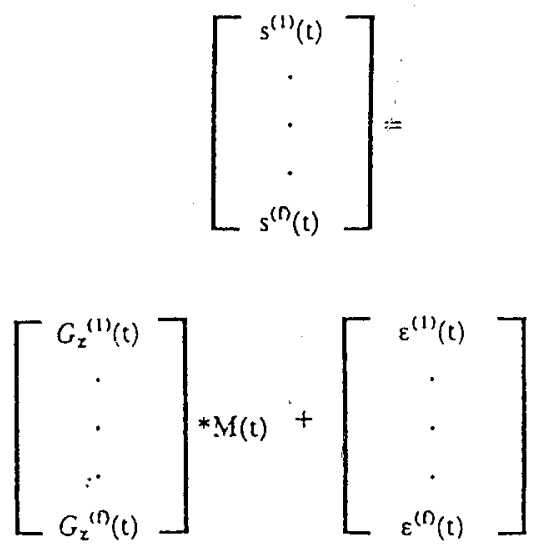

Every seismogram $s^{(i)}(t)$ has its own Green's function $G_{z}{ }^{(i)}(\mathrm{t})$; the asterisk stands for convolution. The noise in the physical system is represented by $\varepsilon$. The moment rate function is generally unknown. A discretized version of the above set of equations is

$$
\overline{\mathrm{d}}=\mathrm{A}_{\mathrm{z}} \cdot \overline{\mathrm{m}}+\overline{\mathrm{\varepsilon}}
$$

where $\overline{\mathrm{m}}=\left(\mathrm{m}_{1}, \ldots, \mathrm{m}_{\mathrm{p}}\right)^{\mathbf{T}}$ is the discretized mo ment rate, $\bar{d}=\left(d_{1}, \ldots, d_{n}\right)^{T}$ a stack of the discretized seismograms $(p<n)$. Matrix $A_{2}$ contains discretized versions of the Green's functions $G_{z}{ }^{(i)}$ and is contracted with $\bar{m}$. It is generally assumed that the components of $\bar{\varepsilon}$ are independently distributed and that their probability distribution is centered around zero. For an assumed depth $\mathrm{z}^{\prime}$, estimating $\overline{\mathrm{m}}$ is a least squares inverse problem and (8) can be solved using standard technqiues [e.g., some generalized inverse. For a given data set we can simply try a range of depths $z^{\prime}$, the best

\section{CHILE 26 SEP 1967}

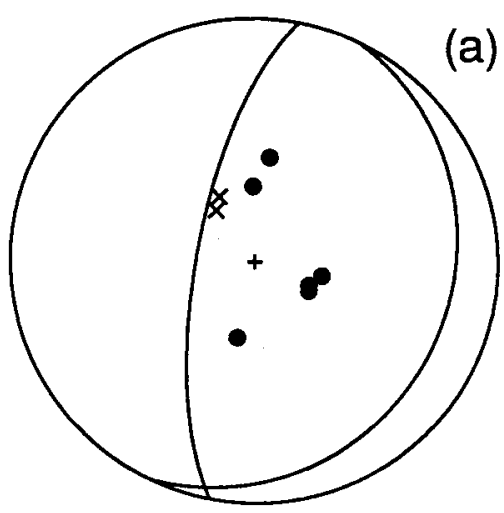

\section{CHILE 4 OCT 1983}

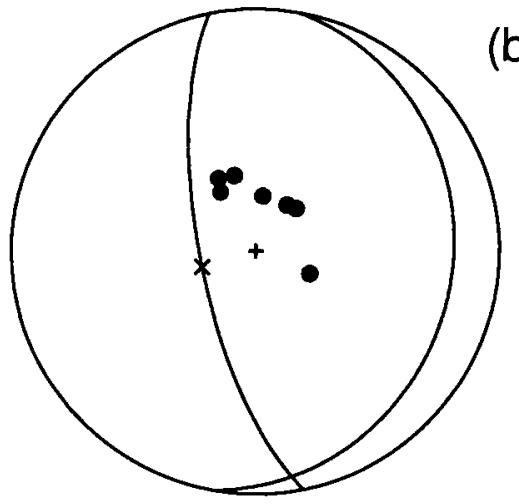

(b)

Fig. 4. Focal mechanism and station distribution for the $(a) 1967$ and $(b) 1983$ earthquake. Shown is the stereographic projection of the focal sphere for both events [e.g, Aki and Richards, 1980]. The solid circles represent stations with a compressional direct $P$ arrival, while the crosses are nodal arrivals. From the orientations of the fault and auxiliary planes (great circles), combined with the epicentral locations, it is known that both earthquakes occurred on the interface between the South American Plate and the subducting Nazca Plate. 
depth given by the case that produces the best fit to the data. In a resampling scheme, for each resampled data vector $\bar{d}^{*}$ the best depth estimate follows from

$$
\begin{gathered}
Z_{\text {est }}^{*}=\min _{z^{\prime}}\left[\bar{e}^{T} \bar{e}\right] \\
\bar{e}=\bar{d}^{*}-A_{z^{\prime}}^{*} \cdot F_{z^{\prime}}^{*-1} \cdot \bar{d}^{*}
\end{gathered}
$$

The parameter $z^{\prime}$ is a very complicated nonlinear function of the data, and it is not possible to theoretically assess whether the jackknife or the bootstrap variance estimator give better results.

As an example, we estimate depth and its standard deviation for two earthquakes by resampling a set of long-period $P$ wave seismograms. We applied both the bootstrap estimator (6) and the "delete-half" jackknife estimator (formula (5) with $\mathrm{k}=\mathrm{n} / 2$ ). The two earthquakes are the September 26, 1967, Coquimbo earthquake $\left(M_{\mathrm{w}}=5.9\right.$, origin time 1611 GMT) in central Chile, and the much larger October 4, 1983, Taltal earthquake $\left(\mathrm{M}_{\mathrm{w}}=7.3\right.$, origin time $\left.0419 \mathrm{GMT}\right)$ in northern Chile. Both seismic events occurred on the interface between the South American Plate and the subducting Nazca Plate. Several previous depth estimates are available for these events: first of all, the International Seismological Center depths are $40 \mathrm{~km}$ and 5 $\mathrm{km}$ for the 1967 and 1983 earthquake, respectively; the Harvard CMT depth for the 1983 event is $39 \mathrm{~km}$; and Malgrange and $\mathrm{Ma}$ dariaga [1983] estimate a depth of $48 \mathrm{~km}$ for the 1967 event. To ensure a good depth resolution, we collected a set of eight seismograms that are well distributed in source-station azimuth for both earthquakes (Figure 4) The results of the jackknife (Figures $5 a, 6 a$ ) and the bootstrap (Figures $5 b, 6 b$ ) are similar and yield a depth of $48 \pm 1 \mathrm{~km}(2 \hat{\sigma})$ for the 1967 earthquake and $26 \pm 11 \mathrm{~km}(2 \hat{\sigma})$ for the 1983 event. For all resampling experiments the total number of resamples was 100 . It is interesting to compare these depths to the data variance as a function of assumed depth for the full data set. The minimum variance depth for the 1967 earthquake (Figure $5 c$ ) is similar to the depth found with the two resampling techniques, and data variance increases rapidly around the minimum, which agrees qualitatively with a small standard error. For the 1983 earthquake the data variance curve is much wider (Figure $6 c$ ) and has several minima, which agrees with a large standard error of the depth. Figure 7 shows the waveforms and the estimated moment rate function (source time function) for both events. The 1967 earthquake has a source duration of $2 \mathrm{~s}$, and the waveforms show the arrival of secondary phases. These phases are the depth phases, and their timing relative to the direct arrival provides an independent way of estimating depth and its standard error. Unlike the direct phase, the depth phase is seismic energy that does not travel directly to the seismographic station, but first travels almost vertically upward from the earthquake's focus to the surface of the Earth and then reflects partly back into the Earth, heading for the station. We measured the relative timing for the eight seismograms, which translates into a depth of $45 \pm 5 \mathrm{~km}(2 \hat{\sigma})$, a depth compatible with the resampling results. The standard error is larger than the jackknife and bootstrap estimate. This is to be expected because the number of data used to measure the relative timing of depth phases
CHILE 26 SEP $1967 \mathrm{M}_{\mathrm{W}}=5.9$
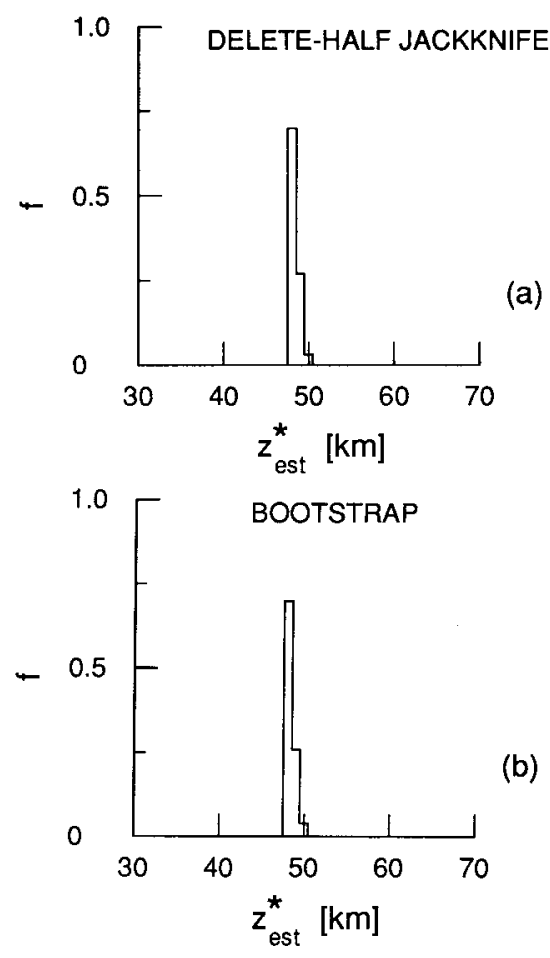

(b)

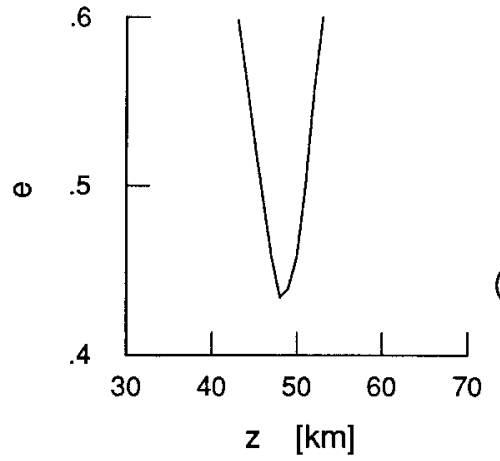

Fig. 5. Results for the $1967 \mathrm{M}_{\mathrm{w}}=5.9$ Coquimbo earthquake in central Chile. The full data set consists of 320 samples, 40 per seismogram. The (a) "delete-half" jackknife depth estimate is $48.3 \pm 1.1 \mathrm{~km}$ $\left(2 \hat{\sigma}_{J A C K}\right)$, which is the same as the $(b)$ bootstrap result. The normalized data variance $\mathrm{e}$ for the full $(c)$ data set has a sharp minimum at $48 \mathrm{~km}$.

is more than a factor of ten smaller than the number of data used in waveform inversion. The 1983 waveforms do not show the secondary depth phase arrivals. Because this large earthquake has a moment rate function with a total duration of more than $30 \mathrm{~s}$, the depth phases arrive while the direct phase is still significantly affecting ground motion at the seismographic station. Thus the relative timing of a depth phase cannot be measured directly from the seismogram. Depth resolution from long-period $P$ waves results completely from the depth phases. For a large earthquake like the 1983 Taltal event, the noise in the depth phase has superimposed on it the noise in the direct phase. Thus it is not surprising that the 1983 depth has a large standard error. Perhaps the most im-
CHILE 4 OCT $1983 \mathrm{M}_{\mathrm{W}}=7.3$
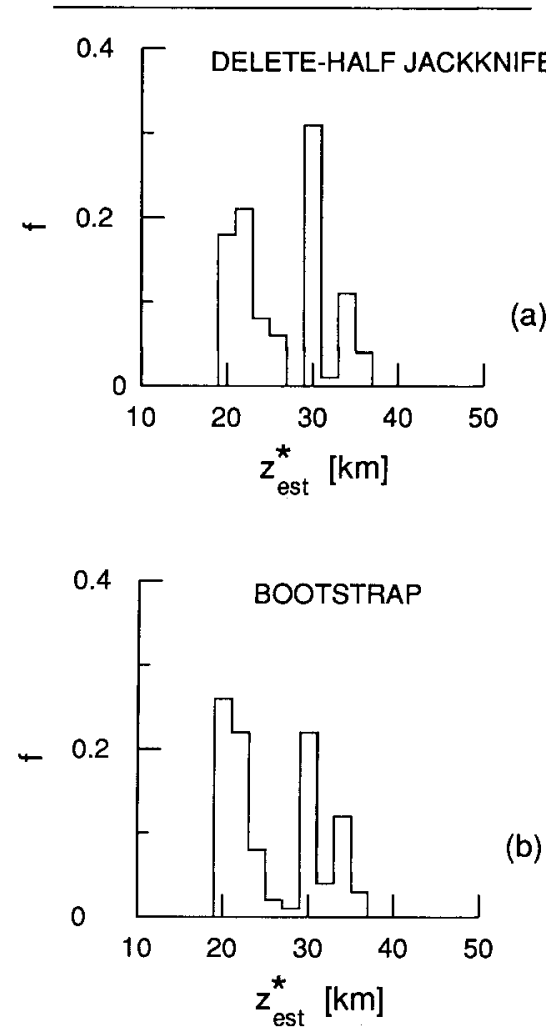

(b)

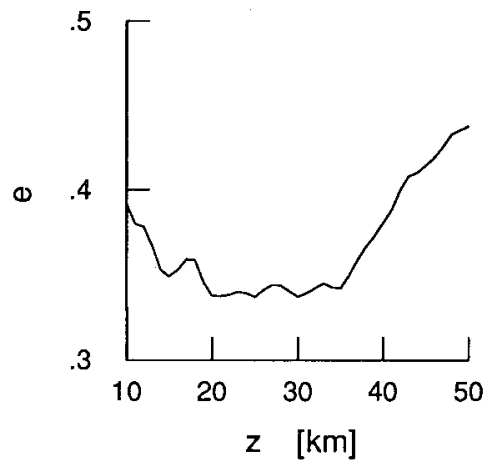

Fig. 6. As Figure 5, but now for the 1983 $\mathbf{M}_{\mathbf{w}}=7.3$ Taltal earthquake in northern Chile. The full data set has 400 samples, 50 per seismogram. The $(a)$ "delete-half" jackknife depth estimate is $26.5 \pm 10.3 \mathrm{~km}$ $\left(2 \hat{\sigma}_{J A C K}\right)$, which is essentially the same as the $(b)$ bootstrap esitmate of $25.8 \pm 10.8$ $\mathrm{km}\left(2 \hat{\sigma}_{B O O T}\right)$. The normalized data variance $\mathrm{e}$ for the $(c)$ full data set has a poorly defined global minimum.

portant conclusion we now state is that jackknifing and bootstrapping "prove" that the 1967 earthquake is resolvably deeper than the 1983 earthquake.

\section{Discussion}

We have introduced the statistical concepts of resampling to a geophysical audience. Two methods, jackknifing and bootstrapping, can be used to estimate model variance. Applica- 


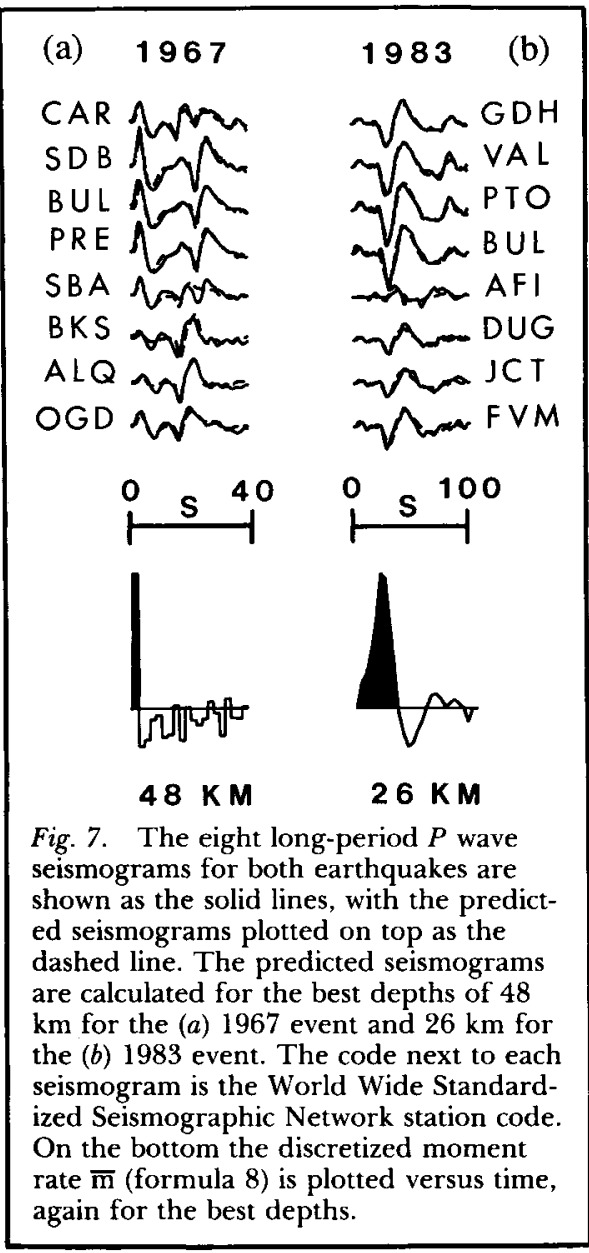

tion of the methods to two Chilean earthquakes yield earthquake depth, as well as its standard error. Resampling techniques are a topic of current research in statistics, and as noted by Diaconis and Efron [1983], they represent a new generation of "computationally intensive" statistical techniques. We believe that resampling techniques offer a way for geophysicists to estimate model variance in complicated geophysical problems.

\section{Acknowledgments}

This research is part of the earthquake research program at the University of Michigan. BWT was supported by a Shell Ph.D. fellowship, and LJR was supported by grants from the National Science Foundation (EAR8351515 and EAR8720935). BWT would like to thank Nalini Bhushan for her enthusiastic philosophical support.

\section{References}

Aki, K., and P. G. Richards, Quantitative Seismology, vol. 1, 558 pp., W. H. Freeman and Company, San Francisco, Calif. 1980.

Diaconis, P., and B. Efron, Computer intensive methods in statistics, Sci. Am., 248, 116 1983.

Efron, B., Computers and the theory of statistics: Thinking the unthinkable, SIAM Rev., 21, 460, 1979.
Efron, B., The Jackknife, the Bootstrap and Other Resampling Plans, 92 pp., SIAM, Philadelphia, Pa., 1982

Efron, B., and G. Gong, A leisurely look at the bootstrap, the jackknife, and cross-Validation, Am. Stat., 37, 36, 1983.

Efron B., and C. Stein, The jackknife estimate of variance, Ann. Stat., 9586, 1981.

Guttorp, P., and A. Walden, On the evaluation of geophysical models, Geophys. J. R. Astron. Soc., 91, 201, 1987.

McLaughlin, K. L., Maximum-likelihood event magnitude estimation with bootstrap ping for uncertainty estimation, Bull. Seismol. Soc. Am., 78, 855, 1988.

Malgrange, M., and R. Madariaga, Complex distribution of large thrust and normal fault earthquakes in the Chilean Subduction Zone, Geophys. J. R. Astron. Soc., 73, 489, 1983.

Menke, W., Geophysical Data Analysis: Discrete Inverse Theory, 260 pp., Academic Press, Orlando, Fla., 1984.

Ruff, L. J., Multi-trace deconvolution with unknown trace scale factors: Omnilinear inversion of $P$ and $S$ Waves for source time functions, Geophys. Res. Lett., in press, 1989.

Tichelaar, B. W., and L. J. Ruff, Rupture extent of the 1978 Miyagi-Oki, Japan, earthquake and seismic coupling in the Northern Honshu Subduction Zone, Geophys. Res. Lett., 15, 1219, 1988.

Willmott, C. J., S. G. Ackleson, R. E. Davis, J J. Feddema, K. M. Klink, D. R. Legates, J. O'Donnell, and C. M. Rowe, Statistics for the evaluation and comparison of models, J. Geophys. Res., 90, 8995, 1985.

Wu, C. F. J., Jackknife, bootstrap, and other resampling methods in regression analysis, Ann. Stat., 14, 1261, 1986.

\section{Energetics and Dynamics of Solar Activity}

\section{Gerard Van Hoven}

Department of Physics, University of California, Irvine

The activity of the Sun occurs in the solar atmosphere and is driven and confined by the Sun's magnetic field. The plasma atmosphere comprises the cool $\left(\leqslant 10^{4} \mathrm{~K}\right)$ and dense chromosphere, an intermediate transition region, and the hot $\left(T_{c} \geq 10^{6} \mathrm{~K}\right)$ and diffuse $\left(n_{c} \geqslant 10^{8} \mathrm{~cm}^{-3}\right)$ corona; the solar atmosphere has been well observed from the ultraviolet through hard $\mathrm{X}$ rays, by the Skylab and Solar Maximum Mission spacecraft, among others. The atmospheric magnetic field has its source in the solar interior and is driven and energized by the global differential rotation and local turbulent motions at the visible photospheric surface; this field is mostly known from Zeeman-effect and microwave measurements. The magnetic field lines return to the surface in active regions and are open in coronal holes that provide the source for the solar wind.
The primary concern of the Solar Plasma Theory Group at the University of California, Irvine, involves the dynamics and energetics of magnetic activity. The coronal field is strong $\left(B^{2} / 2 \mu_{0} P>>1\right)$, nonuniform, and stressed by currents driven from the surface. This field provides a source of stored energy and an anisotropic medium for the channeling of mass and energy flows in the atmospheric plasma.

There are a number of unsolved problems connected with observed solar activity. They are briefly stated (to be amplified in the following sections) here. How is the corona heated; that is, how is the necessary energy transported up the adverse temperature gradient from the photospheric source surface and deposited in the corona, apparently most effectively in strong-field regions? How does the atmospheric magnetic field channel and confine the ambient heat and mass flows so that the former are suppressed, allowing runaway radiation losses to occur, and the latter are abetted so that the cool condensation of a solar prominence is formed? How can the stressed magnetic field of an active region be reconnected quickly enough, in the highly conducting corona, to explain the short time scales of a solar flare? How are coronal mass ejections launched against the gravitational and magnetic forces of the lower atmosphere so that they can escape into the solar wind?

The UCI group has made significant progress on each of these problems, as will be described in the following sections. The key to our attack on the coupled dynamics and energetics of such nonuniform, anisotropic, nonlinear, active phenomena is the application of large-scale numerical simulations, balanced and supported by analytic calculations and perturbation computations.

\section{Coronal Heating}

One of the outstanding mysteries of solar physics is how the corona is able to sustain its high temperature while the chromosphere below, separated only by a narrow transition region, has a temperature two orders of magnitude lower. Energy must be transported from the solar surface to the corona by mechanisms other than thermal conduction, or large-scale convection, which is not present in the transition region.

Because the corona is threaded by magnetic fields of various intensities, Alfven waves provide a promising medium of energy trans- 